\title{
A phenomenological model for the Meyer-Neldel rule
}

\author{
Jeppe C Dyre \\ Institute of Mathematics and Physics, Imfufa, University of Roskilde, Postbox 260, \\ DK-4000 Roskilde, Denmark
}

Received 18 November 1985, in final form 24 April 1986

\begin{abstract}
In this paper it is argued that the generality of the Meyer-Neldel rule compels one to adopt a phenomenological approach if a universally valid model is aimed at. It is shown that there exists only one possible phenomenological model. This model is based on an exponential probability distribution of energy barriers. The model predicts a power-law frequency dependence of the AC conductivity with the exponent $s$ given by $s=1-T / T_{0}$ where $T_{0}$ is the characteristic temperature of the Meyer-Neldel rule. It is conjectured that the exponential energy barrier distribution derives from a 'glass transition' at $T_{0}$. The generalisation of the model to account for the compensation effect in other contexts is briefly discussed, using the case of heterogeneous catalysis as an example.
\end{abstract}

\section{Introduction}

Semiconductors are characterised by the temperature dependence of their electrical conductivity. If $\sigma(0)$ denotes the DC conductivity one usually writes

$$
\sigma(0)=A \mathrm{e}^{-\Delta E / k T}
$$

where $\Delta E$ is the activation energy, $k$ is the Boltzmann constant, $T$ is the temperature, and $A$ is the so-called pre-exponential factor. Almost 50 years ago, Meyer and Neldel (1937) found for some oxide semiconductors that when the semiconductor is prepared or annealed under different conditions, the activation energy varies and that $A$ depends exponentially on $\Delta E$. Their discovery has become known as the Meyer-Neldel (MN) rule. It states that the $D C$ conductivity is given by

$$
\sigma(0)=\sigma_{00} \mathrm{e}^{\Delta E / k T_{0}} \mathrm{e}^{-\Delta E / k T}
$$

where $\sigma_{00}$ and $T_{0}$ are constants within a class of related semiconductors.

The MN rule is an empirical relation which has been observed in very many different semiconductors. These include single-crystal and polycrystalline semiconductors, amorphous semiconductors, organic semiconductors, and even ionically conducting crystals and glasses (Rosenberg et al 1968, Roberts 1971, Carlson and Wronski 1979, Dosdale and Brook 1983). The rule applies to chemically closely related semiconductors or to a single semiconductor prepared in various ways. For instance one may vary the degree of non-stoichiometry, the dopant concentration, the oxygen partial pressure at annealing, etc. The MN rule seems to be an almost universal characteristic of semiconductors which have in common only the property of being inhomogeneous from 
some point of view (Irsigler et al 1983). But the relation is even more general than that. In a number of rate processes it is found that the pre-exponential factor $A$ of the reaction rate depends itself exponentially on the activation energy: $A \propto \exp \left(\Delta E / k T_{0}\right)$, just as in (2). This phenomenon is usually referred to as the compensation effect, but it has also been called the Constable law, the isokinetic relationship or the $\theta$-rule (Exner 1964, Khait 1983). A thoroughly studied example is the compensation effect in heterogeneous catalysis which has been reviewed by Galwey (1977). The compensation effect has also been observed by e.g. thermionic emission of electrons from a metal (Vanselow 1985), diffusion in solids (Dienes 1950, Shinar et al 1984), annealing time of irradiated silicon (Fang 1969), viscosity of aqueous solutions (Good and Stone 1972), decomposition of solids (Zsako et al 1981), and a number of biophysical, biochemical and biological systems (Rosenberg et al 1971, Tomlinson 1983).

In this paper the starting point is the observation that one is forced to adopt a phenomenological approach if the many occurrences of the MN rule are believed to have a single common origin. The occurrence of the compensation effect in other contexts even more emphasises the need for a phenomenological approach. While the paper is almost exclusively about the MN rule in semiconductors, the model to be proposed can be generalised immediately to deal with the compensation effect in other rate processes, as will be briefly commented upon in $\S 6$.

The paper is organised as follows. Section 2 argues for the need for a phenomenological model and derives the basic feature of such a model, an exponential probability distribution of energy barriers. In $\S 3$ the simple mathematics of the model is developed. It will be shown that the model predicts a correlation between the MN rule and the AC properties of the semiconductors. In $\S 4$ the model predictions are compared to experiment. In $\S 5$ it is conjectured that the exponential energy barrier distribution is a consequence of a glass transition. Section 6 deals with the generalisation of the model to account for the compensation effect in other rate processes, exemplified by the case of heterogeneous catalysis. A final discussion is given in $\S 7$.

\section{The phenomenological approach}

Several models have been proposed to explain the MN rule. Busch (1950) suggested that the $\mathrm{MN}$ rule in extrinsic broad band semiconductors is due to a freezing of the donor concentration during cooling after the preparation. The MN rule in amorphous or polycrystalline semiconductors may derive from an exponential tailing of the majority band states as suggested by Roberts (1971) and by Cohen et al (1984), or it may be due to a long-ranged electrostatic random potential (Overhof 1984). As regards the $\mathrm{MN}$ rule in organic semiconductors, Kemeny and Rosenberg (1970) proposed a model where electrons or polarons tunnel through intermolecular barriers from activated energy states of the organic molecules. For ionic conductors it has been argued that the MN rule is an approximate relation valid for ionic crystals with either Frenkel, Schottky or interstitial disorder (Dosdale and Brook 1983). It has even been suggested that the MN rule may be a spurious effect due to a thin rectifying layer at the electrode-solid interface (Roberts 1971).

It is of course perfectly possible that the MN rule has many different explanations, each valid for only a limited class of solids. But in view of the generality of the phenomenon it seems more likely that there is a single, general explanation. However, if a single model of the MN rule is to be universally valid, it can not relate directly to the microscopic 
details of the conduction process; it must be a phenomenological model. This is an important, though obvious, conclusion. It will be shown below that once the phenomenological approach is accepted there is only one possible model.

Proceeding now to derive the basic feature of a phenomenological model for the $\mathrm{MN}$ rule, we recall that the rule has been observed in both electronically, polaronically and ionically conducting solids. The only way to describe these different cases by a single model is to use a hopping model, i.e. to regard the conduction process as mediated by hopping of localised charge carriers (to be referred to as 'quasi-particles'). In order to arrive at the simplest possible model, the quasi-particles will be assumed to be noninteracting. Thus, all many-body effects and effects of Fermi statistics are ignored. This is the basic assumption of the present model and it is because of this assumption the model is referred to as 'phenomenological'. Now, the single common feature of the many occurrences of the MN rule is an element of disorder from some point of view (Dewsberry 1975, Irsigler et al 1983). Disorder can easily be built into a hopping model via a probability distribution $p(\tau)$ of waiting times $\tau$ at each quasi-particle site (Scher and Lax 1973). It is known from AC conductivity measurements on numerous disordered semiconductors that there is always a maximum waiting time $\tau_{\max }$, corresponding to the frequency below which the conductivity becomes frequency independent. On the other hand, there is no minimum waiting time $\tau_{\min }$ of any relevance to this problem (i.e. $\tau_{\min } \simeq$ $10^{-12} \mathrm{~s}$ ). Thus, in the phenomenological model a solid is completely characterised by the two entities: $p(\tau)$ and $\tau_{\max }$. A class of $\mathrm{MN}$-related solids must have something in common. It is physically obvious that the DC conductivity depends strongly on $\tau_{\max }$ so the common entity must be $p(\tau)$.

Having reached the conclusion that different MN-related solids have the same $p(\tau)$ but different $\tau_{\max }$, the next step is to ask what can be learned about $p(\tau)$ from (2)-the Meyer-Neldel rule. The DC conductivity is proportional to the diffusion constant which by Einstein's equation is proportional to the mean-square displacement per unit time of a quasi-particle. The DC conductivity is thus proportional to $\lim _{N \rightarrow x} N / \tau_{N}$ where $\tau_{N}$ is the time taken for $N$ jumps, that is

$$
\sigma(0) \propto\langle\tau\rangle^{-1}
$$

where \langle\rangle denotes an average over $p(\tau)$ (all jump-jump correlations are ignored). The quasi-particle jumps are thermally activated so $\tau$ is determined by an energy barrier $\Delta E^{\prime}$

$$
\tau=\tau_{0} \mathrm{e}^{\Delta E^{\prime} / k T}
$$

where $\tau_{0}$ is a microscopic time $\left(\tau_{0} \simeq 10^{-12} \mathrm{~s}\right)$. The maximum waiting time $\tau_{\max }$ corresponds to a maximum energy barrier $\Delta E_{\mathrm{r}}$, while the minimum energy barrier is approximately equal to zero because $\tau_{\min } \simeq \tau_{0}$. If the distribution of energy barriers corresponding to $p(\tau)$ is denoted by $p\left(\Delta E^{\prime}\right)$ we now find by combining (2), (3) and (4)

$$
\mathrm{e}^{-\Delta E / k T_{0}} \mathrm{e}^{\Delta E / k T} \propto \int_{0}^{\Delta E_{\tau}} \mathrm{e}^{\Delta E^{\prime} / k T} p\left(\Delta E^{\prime}\right) \mathrm{d}\left(\Delta E^{\prime}\right)
$$

which is valid for each sample, i.e. for varying $\Delta E$. Taking the derivative of this equation with respect to $\Delta E$ one obtains

$$
\mathrm{e}^{-\Delta E / k T_{0}} \mathrm{e}^{\Delta E / k T} \propto \mathrm{e}^{\Delta E_{\mathrm{\tau}} / k T} p\left(\Delta E_{\tau}\right) \frac{\mathrm{d} \Delta E_{\tau}}{\mathrm{d} \Delta E} .
$$

The constant of proportionality depends weakly on $T$ and $\Delta E_{\tau}$ but this is unimportant compared to the exponentials and it will be ignored. By considering the temperature 
dependence of both sides of (6), one finds that $\Delta E=\Delta E_{\tau}$. This is a manifestation of the above-mentioned fact that $\sigma(0)$ and $\tau_{\max }$ are strongly correlated. Equation (6) now becomes

$$
p\left(\Delta E^{\prime}\right) \propto \mathrm{e}^{-\Delta E^{\prime} / k T_{0}} .
$$

Thus, once the phenomenological approach is accepted, one is led to a unique model for the MN rule based on an exponential distribution of energy barriers. The exponential energy barrier distribution is well known. It has been used, e.g. for describing AC conductivity of hollandite (Bernasconi et al 1979), dispersive transport in a-Si: $\mathrm{H}$ (Hvam and Brodsky 1981), structural relaxation of amorphous germanium (Olsen 1986), and dielectric polarisation phenomena in glasses (Thurzo et al 1975). A brief review of the history of the exponential energy barrier distribution has been given by Macdonald (1985).

\section{The model}

It is well known that variations in quasi-particle jump distance are of little importance compared to the large variations in waiting times. This fact may be built into the model by adopting the Scher-Lax approach (1973) and regarding the disordered semiconductor as a regular lattice with randomly varying jump frequencies, and this approach is adopted here. It is convenient to speak of jump frequencies $\gamma$ instead of waiting times, these two quantities are related by $\gamma=\tau^{-1}$ so the jump frequency is given by

$$
\gamma=\gamma_{0} \mathrm{e}^{-\Delta E^{\prime} / k T}
$$

where $\gamma_{0}$ is the attempt frequency $\left(\simeq 10^{12} \mathrm{~s}^{-1}\right)$ and $\Delta E^{\prime}$ is the energy barrier.

As mentioned, the MN rule is observed in disordered semiconductors. This is the same class of solids that is known to show strong frequency dispersion of the conductivity, and therefore it is of interest to evaluate the entire frequency-dependent conductivity of the model, $\sigma(\omega)$. This quantity can be calculated by the CTRW approximation, the simplest possible non-trivial mean-field approximation. In the CTRW $\sigma(\omega)$ is given by (Scher and Lax 1973, Odagaki and Lax 1981)

$$
\sigma(\omega)=\frac{C}{T}\left[-\mathrm{i} \omega+\left\langle\frac{1}{\gamma+\mathrm{i} \omega}\right\rangle^{-1}\right]
$$

where \langle\rangle denotes an average over the jump frequency probability distribution $p(\gamma)$, and $C$ is a constant depending on the quasi-particle concentration, average jump distance, etc.

The jump frequency distribution is really a probability density: $p(\gamma) \equiv \mathrm{d} \tilde{p} / \mathrm{d} \gamma$. From this we find by means of (7) and (8) (Blumen et al 1986)

where

$$
p(\gamma)=\frac{\mathrm{d} \tilde{p}}{\mathrm{~d} \gamma}=\frac{\mathrm{d} \tilde{p}}{\mathrm{~d} \Delta E^{\prime}} \frac{1}{\left(\mathrm{~d} \gamma / \mathrm{d} \Delta E^{\prime}\right)} \propto \mathrm{e}^{-\Delta E^{\prime} / k T_{0}} \frac{1}{\gamma} \propto \gamma^{-s}
$$

$$
s=1-T / T_{0} .
$$

The maximum jump frequency is $\gamma_{0}$ (corresponding to $\Delta E_{\min }^{\prime}=0$ ) while the minimum jump frequency is given by

$$
\gamma_{\min }=\gamma_{0} \mathrm{e}^{-\Delta E / k T}
$$


where $\Delta E$ as in $\S 2$ is the maximum energy barrier $\left(=\Delta E_{\tau}\right)$. The normalised jump frequency distribution is given by

$$
p(\gamma)=\frac{1-s}{\gamma_{0}}\left(\frac{\gamma}{\gamma_{0}}\right)^{-s}
$$

Here it has been assumed that $\gamma_{\min } \ll \gamma_{0}$ which is valid in all cases of interest and will be assumed throughout the paper. Also, $s>0$ will be assumed. This corresponds to the temperature range of interest in most experiments, namely $T<T_{0}$.

Proceeding now to calculate the conductivity we find for the DC conductivity

$$
\sigma(0)=\frac{C}{T}\left\langle\gamma^{-1}\right\rangle^{-1}=\frac{C}{T} \frac{s}{1-s} \gamma_{0}\left(\frac{\gamma_{\min }}{\gamma_{0}}\right)^{s}
$$

or

$$
\sigma(0)=\frac{C}{T} \frac{s}{1-s} \gamma_{0} \mathrm{e}^{\Delta E / k T_{0}} \mathrm{e}^{-\Delta E / k T} .
$$

As expected we recover the $\mathrm{MN}$ rule. There is a factor of $T^{2}$ in the denominator differing from (2), but this is almost unobservable compared to the strong temperature dependence of the Boltzmann factor. The AC conductivity is independent of frequency for $\omega \ll \gamma_{\min }$ and equal to $\sigma(0)$. For frequencies around $\gamma_{\min }$ the conductivity begins to increase. In the limit $\omega \gg \gamma_{\min }$ (but still $\omega \ll \gamma_{0}$ ) it is easy to calculate $\sigma(\omega)$. In this regime one finds

$$
\left\langle\frac{1}{\gamma+\mathrm{i} \omega}\right\rangle \simeq \frac{1-s}{\gamma_{0}} \int_{0}^{\infty} \frac{\left(\gamma / \gamma_{0}\right)^{-s}}{\gamma+\mathrm{i} \omega} \mathrm{d} \gamma=\frac{1}{\gamma_{0}} \frac{(1-s) \pi}{\sin (s \pi)}\left(\frac{\mathrm{i} \omega}{\gamma_{0}}\right)^{-s}
$$

and since the $\mathrm{i} \omega$-term of (9) is negligible, $\sigma(\omega)$ is simply given by

$$
\sigma(\omega)=\frac{C}{T} \gamma_{0} \frac{\sin (s \pi)}{(1-s) \pi}\left(\frac{\mathrm{i} \omega}{\gamma_{0}}\right)^{s} \quad \gamma_{\min } \ll \omega \ll \gamma_{0} .
$$

Thus a power-law frequency dependence of the conductivity results, with the exponent $s=1-T / T_{0}$. It must be emphasised, however, that $\sigma(\omega) \propto \omega^{s}$ with $s=1-T / T_{0}$ is a direct consequence of (13); the CTRW approximation really only enters in the calculation of the constant of proportionality. The evaluation of $\sigma(\omega)$ for all $\omega$ has been discussed by Macdonald (1985).

\section{The phenomenological model and the experimental situation}

As shown above, the phenomenological model implies a power-law frequency dependence of the AC conductivity. This is a striking prediction which can be easily checked experimentally, it seems. And indeed, all disordered semiconductors exhibit a $\omega^{s}$ behaviour of the AC conductivity where $s$ is always less than one and always tends to one as $T$ tends to zero (Jonscher 1977, Long 1982). Still, these experimental findings can not be taken as evidence for the model. The reason is the following. For most solids $\Delta E /$ $k T_{0}$ is not very much larger than one and therefore it is necessary to go to temperatures well below $T_{0}$ in order to observe a power-law behaviour of $\sigma(\omega)$ covering a wide frequency range. But at low temperatures, the above model is hard to distinguish from another phenomenological model which has nothing to do with the MN rule. This model, 
which corresponds to the $T_{0} \rightarrow \infty$ and $\gamma_{0} \rightarrow \infty$ limit of the MN-rule model, predicts that (Dyre 1985a)

$$
\sigma(\omega)=\sigma(0) \frac{\mathrm{i} \omega \tau}{\ln (1+\mathrm{i} \omega \tau)}
$$

where $\tau=\gamma_{\min }^{-1}$. This formula implies an AC conductivity which is very close to a power law $\sim \omega^{s}$ where $s$ at low temperatures is given by (Dyre 1985b)

$$
s=1-T / T_{1} \quad k T_{1}=\frac{1}{2} \Delta E .
$$

Here $\Delta E$ as usual denotes the activation energy of $\sigma(0)$. Obviously, accurate measurements of the AC conductivity are necessary in order to distinguish the $\mathrm{MN}$-rule model from the model behind (18).

It seems that few measurements of $\sigma(\omega)$ have been carried out on the same samples that have been reported to obey the MN rule. Searching the literature, the author only found one example of this. In an $\mathrm{Ag}$ ionically conducting glass the exponent $s$ is equal to 0.7 at $T=119 \mathrm{~K}$ and at $T=134 \mathrm{~K}$ (Almond et al 1985). This is consistent with (11) because the Meyer-Neldel temperature $T_{0}$ of this glass is $450 \mathrm{~K}$.

\section{The Meyer-Neldel rule as a consequence of a glass transition}

In this section the origin of the MN rule is discussed. First one may ask whether $T_{0}$ is to be interpreted as a real physical temperature or whether $k T_{0}$ is just a characteristic energy. The latter point of view is held by the workers postulating exponential band tails (Roberts 1971, Adler 1982, Cohen et al 1984) and by Kemeny and Rosenberg (1970) who link $T_{0}$ to the Debye temperature which really is an energy scale. Here, we shall suggest that $T_{0}$ is a real physical temperature, namely a glass transition temperature.

Remember that the MN rule implies that the DC conductivity at $T=T_{0}$ is independent of $\Delta E$. According to the phenomenological model, as $T$ rises towards $T_{0}$ the deepest energy minima become less important for the determination of $\sigma(0)$ and right at $T=T_{0}$ they are not important at all. This is reflected by the fact that $p(\gamma) \propto \gamma^{-s}$ becomes $p(\gamma)=$ constant at $T=T_{0}$. When the deepest energy minima lose their importance it is obvious that $\sigma(0)$ becomes independent of $\Delta E$, the maximum energy barrier. In the $\Delta E^{\prime} \rightarrow 0$ limit, energy maxima and energy minima are indistinguishable so the population of energy maxima becomes non-vanishing at $T=T_{0}$. This means that the picture of activated conductivity breaks down in the setting of an exponential energy barrier distribution, so that the model does not apply for $T>T_{0}$.

What happens at $T_{0}$ is hinted at by the exponential energy barrier distribution itself. Since on the average $\Delta E^{\prime}=$ constant $-E^{\prime}$, where $E^{\prime}$ is the energy of the state which has the barrier $\Delta E^{\prime}$ to its surrounding states, we find immediately from (7)

$$
n\left(E^{\prime}\right) \propto \mathrm{e}^{E^{\prime} / k T_{0}}
$$

where $n\left(E^{\prime}\right)$ is the density of energy levels. It must be emphasised that (20) refers to the quasi-particle-lattice interaction energy and does not include any quasi-particle 'kinetic energy'. A macroscopic system with the density of energy levels given by (20) undergoes a phase transition at $T=T_{0}$ to a state of zero entropy (a Gibbs-DiMarzio glass transition (Gibbs and DiMarzio 1958)). The system is 'frozen' into its lowest lying energy levels. A quasi-particle is only a microscopic system, but still (20) implies that the quasi-particle 
energy fluctuations decrease drastically below $T_{0}$. A guess of what happens at $T_{0}$ is that the relevant lattice degrees of freedom freeze, so that the quasi-particles for $T<T_{0}$ are unable to exchange potential energy with the lattice. By freezing, the crystallisation of the liquid state is not necessarily meant. In general, it could be a freezing-in of the various kinds of lattice defects that determine the quasi-particle energy. When the lattice freezes, the quasi-particle-lattice interaction energy $E^{\prime}$ becomes a function of the quasi-particle coordinates only. This function is more or less random in space and (20) then just expresses the probability of finding energy $E^{\prime}$ at a given quasi-particle position. In summary, it is conjectured that the MN rule and (20) are consequences of a frustration of thermal equilibrium at $T_{0}$. The frustration takes place on cooling at the sample preparation. If this picture is correct, the element of disorder common to all occurrences of the MN rule simply derives from a freezing of thermal disorder at $T_{0}$.

A frustration of thermal equilibrium can be referred to as a glass transition. The concept is here used in a generalised sense, often one only speaks of a glass transition in connection with the solidification of supercooled liquids. The idea that the MN rule is due to a glass transition, is not new. Busch (1950) suggested that the MN rule for extrinsic semiconductors derives from a freezing of the donor concentration during cooling after the sample preparation (Metselaar and Oversluizen 1984). This idea has been generalised here to the conjecture that the MN rule universally is a consequence of a glass transition. It must be emphasised, however, that at present this is only a conjecture.

\section{Generalisation of the model. A model for the compensation effect in heterogeneous catalysis}

The topic of this section is the generalisation of the phenomenological model to deal with the compensation effect in contexts other than electrical conductivity. By the compensation effect is meant the phenomenon that the reaction rate $K$ for a class of related rate processes is given by

$$
K=\text { constant } \times \mathrm{e}^{\Delta E / k T_{0}} \mathrm{e}^{-\Delta E / k T} .
$$

In this more general setting $T_{0}$ is often referred to as the isokinetic temperature. To be specific we shall discuss only the compensation effect in heterogeneous catalysis (Cremer 1955, Sinfelt 1969, Galwey 1977), but it is straightforward to generalise the considerations below to deal with other cases. First, a note of caution: There is considerable evidence that the great majority of reported regularities following (21) is in error and has arisen only by an incorrect treatment of data (Exner 1973, Krug et al 1976). The compensation effect in chemistry is therefore a less mature subject than the MN rule in physics, which is firmly established. However, there is agreement in the literature that genuine examples of compensating systems also exist in chemistry. It is these systems to which the model described below applies.

In the present model heterogeneous catalysis is regarded as the result of many possible reaction sequences, each sequence consisting of many intermediate steps. The intermediate states are situated at local energy minima in the abstract reaction coordinate space and an intermediate reaction is a thermally activated 'jump' between two adjacent minima. This is analogous to quasi-particle jumps in ordinary space and the overall reaction rate $K$ becomes the analogue of $\sigma(0)$. Because of the complexity in the model, a statistical approach is needed for the calculation of $K$. Analogously to the MN-rule case it can now be shown that an exponential energy barrier distribution implies (21). 
As in $\S 5$, the exponential distribution is conjectured to derive from a glass transition at $T_{0}$. The glass transition is here the freezing of the relevant catalyst surface degrees of freedom-they determine the energy function in reaction coordinate space.

If this model is correct there is no such thing as the mechanism of a heterogeneous catalytic reaction. Many different reaction sequences contribute to the overall reaction, and the whole thing is so complex that a statistical approach is necessary. Actually, the complexity of heterogeneous catalysis is commonly recognised to the extent that the mechanism of catalysis is often referred to as 'black magic'. Once the statistical approach is accepted, however, the argumentation of $\S 2$ applies from which it can be concluded that the model is unique in the sense that only an exponential energy barrier distribution is possible if the compensation effect is to be reproduced (assuming $\Delta E_{\min }^{\prime}=0$ ).

The model implies that interesting time-dependent phenomena must be present in connection with the compensation effect. As a consequence of the exponential energy barrier distribution, it is predicted that a power-law, time or frequency dependence should be observed in chemical relaxation experiments with an exponent $s$ given by (11).

\section{Summary and discussion}

The MN rule is a universal phenomenon and this fact emphasises the need for a phenomenological model. As shown in $\S 2$, once the phenomenological approach is accepted there is only one possible model, namely that based on an exponential distribution of energy barriers. The exponential barrier distribution is well known; an early study of it was given by Macdonald (1963). And the idea of deriving the MN rule from a distribution of energy barriers goes back to Henisch (1951), who argued that a gaussian distribution of energy barriers leads to a behaviour in qualitative agreement with the $\mathrm{MN}$ rule. The phenomenological model thus contains few new ideas; it is just an attempt to present a coherent picture of conductivity in MN semiconductors. Though the model was constructed to explain the DC properties, it implies a perhaps surprising prediction of a correlation between the MN rule and the AC properties: $\sigma(\omega) \propto \omega^{s}$ where $s=1-T / T_{0}$ $(\S 3)$.

The experimental situation is complex and there exists no conclusive evidence in favour of the model. The $\mathrm{MN}$ rule is observed in disordered semiconductors, exactly the same class of solids that is known to show a power-law frequency dependence of the AC conductivity. This, and the fact that the exponent $s$ always obeys $s<1$ and $s \rightarrow 1$ for $T \rightarrow 0$, is in agreement with model predictions, but it cannot be taken as evidence for the model as discussed in $\S 4$. Careful experiments are needed, for instance measuring $s(T)$ as $T \rightarrow 0$.

In $\S 5$ it was suggested that the $\mathrm{MN}$ rule is a consequence of a glass transition. It was conjectured that the exponential energy barrier distribution follows from a freezing-in of the lattice degrees of freedom defining the quasi-particle energy, i.e. of the various relevant lattice defects. In this picture both the $\mathrm{MN}$ rule and the conductivity dispersion are ultimately consequences of broken ergodicity (Palmer 1982). The previously mentioned measurements on an Ag ionically conducting glass by Almond et al (1985) fit nicely into this yet somewhat speculative idea; here the $\mathrm{MN}$ temperature $T_{0}$ is equal to the melting temperature of the glass, i.e. the glass transition temperature.

At $T=T_{0}$ the phenomenological model breaks down and a kind of a phase transition is expected. In particular, the conductivity becomes frequency independent at this temperature. Usually it is not possible to measure at this high temperature. Some 
experiments at $T_{0}$ have been carried out, though, and there are signs of something happening at $T_{0}$ as signalled by a small discontinuous change of activation energy (figure 1 of Weichman and Kužel (1970), figure 1 of Irsigler et al (1983)). Further experiments near $T_{0}$ would be very interesting.

The MN rule is only one manifestation of the very general compensation effect. The need for a universal explanation is increasingly realised, and this problem has been addressed by several authors recently suggesting various modifications of rate theory (Conner 1982, Peacock-López and Suhl 1982, Khait 1983, McCoy 1984). In this paper rate theory has been assumed to apply. It is straightforward to generalise the phenomenological model to deal with the compensation effect in general, as was discussed in $\S 6$ exemplified by the case of heterogeneous catalysis. Quasi-particle jumps in ordinary space are replaced by reactions in the abstract reaction coordinate space, but the exponential energy barrier distribution remains unchanged. The basic assumptions of the generalised model are: (i) the existence of an element of disorder, and (ii) that the overall reaction is the result of many reaction sequences acting in parallel, each sequence involving many intermediate steps. The reaction sequences may differ in their intermediate states, or they may involve the same intermediate states where, however, the precise energetics of the reaction path alter (Bond 1985). In the model the overall reaction rate becomes the analogue of the DC conductivity of the MN rule, and the uniqueness of the generalised model can be proved just as in $\S 2$.

As in $\S 5$ it may be argued that a glass transition at $T_{0}$ is the origin of the exponential energy barrier distribution of the generalised model. It is interesting that the compensation effect almost always involves solids. These solids must contain an element of disorder deriving from a freezing of thermal disorder at $T_{0}$, if the ideas of $\$ 5$ are correct. As regards the observation of the compensation effect in liquid solutions (Leffler 1955), it is hard to see how the glass transition idea can apply, though.

The generalised model predicts a power-law time dependence of chemical relaxation experiments and also that a kind of phase transition must occur at $T_{0}$. It would be very interesting to have these predictions tested experimentally.

\section{Acknowledgments}

The author gratefully acknowledges inspiration and encouragement from N B Olsen and several useful improvements of the manuscript suggested by Professor J R Macdonald.

\section{References}

Adler D 1982 Sol. Energy Mater. 853

Almond D P, Duncan G K and West A R 1985 J. Non-Cryst. Solids $\mathbf{7 4} 285$

Bernasconi J, Beyeler H U, Strässler S and Alexander S 1979 Phys. Rev. Lett. 42819

Blumen A, Klafter J and Zumofen G 1986 Fractals in Physics ed. L Pietronen

Bond G C 1986 Z. Phys. Chem. 14421

Busch G 1950 Z. Angew. Math. Phys. 181

Carlson D E and Wronski C R 1979 Amorphous Semiconductors, Topics in Applied Physics vol 36, ed. M H Brodsky (New York: Springer) p 287

Cohen M H, Economou E N and Soukoulis C M $1984 J$ J. Non-Cryst. Solids 66285

Conner W C 1982 J. Catal. 78238

Cremer E 1955 Adv. Catal. 775

Dewsberry R 1975 J. Phys. D: Appl. Phys. 81797 
Dienes G J 1950 J. Appl. Phys. 211189

Dosdale T and Brook R J 1983 Solid State Ionics 8297

Dyre J C 1985a Phys. Lett. 108A 457

- 1985b J. Physique Coll. 46 C8 343

Exner O 1964 Nature 201488

1973 Prog. Phys. Org. Chem. 10411

Fang P H 1969 Phys. Lett. 30A 217

Galwey A K 1977 Adv. Catal. 26247

Gibbs J H and DiMarzio E A 1958 J. Chem. Phys. 28373

Good W and Stone J 1972 Electrochim. Acta 171813

Henisch H K 1951 Z. Phys. Chem. 19841

Hvam J M and Brodsky M H 1981 Phys. Rev. Lett. 46371

Irsigler P, Wagner D and Dunstan D J 1983 J. Phys. C: Solid State Phys. 166605

Jonscher A K 1977 Nature 267673

Kemeny G and Rosenberg B 1970 J. Chem. Phys. 533549

Khait Y L 1983 Phys. Rep. 99237

Krug R R. Hunter W G and Grieger R A 1976 J. Phys. Chem. 802335,2341

Leffler J E $1955 \mathrm{~J}$. Org. Chem. 201202

Long A R 1982 Adv. Phys. 31553

McCoy B J 1984 J. Chem. Phys. 803629

Macdonald J R 1963 J. Appl. Phys. 34538

- 1985 J. Appl. Phys. 58 1955, 1971

Metselaar R and Oversluizen G 1984 J. Solid State Chem. $\mathbf{5 5} 320$

Meyer W and Neldel H 1937 Z. Techn. Phys. 12588

Odagaki T and Lax M 1981 Phys. Rev. B 245284

Olsen N B 1986 unpublished

Overhof H $1984 J$. Non-Cryst. Solids 66261

Palmer R G 1982 Adv. Phys. 31669

Peacock-López E and Suhl H 1982 Phys. Rev. B 263774

Roberts G G 1971 J. Phys. C: Solid State Phys. 43167

Rosenberg B, Bhowmik B B, Harder H C and Postow E 1968 J. Chem. Phys. 494108

Rosenberg B, Kemeny G, Switzer R C and Hamilton T C 1971 Nature 232471

Scher H and Lax M 1973 Phys. Rev. B 74491

Shinar J. Davidov D and Shaltiel D 1984 Phys. Rev. B 306331

Sinfelt J H 1969 Catal. Rev. 3175

Thurzo I, Barančok D, Doupovec J, Mariani E and Janči J 1975 J. Non. Cryst. Solids 18129

Tomlinson E 1983 Int. J. Pharm. 13115

Vanselow R 1985 Surface Sci. 149381

Weichman F L and Kužel R 1970 Can. J. Phys. 4863

Zsako J, Varhelyi M, Varhelyi C and Liptay G 1981 Thermochim. Acta 51277 